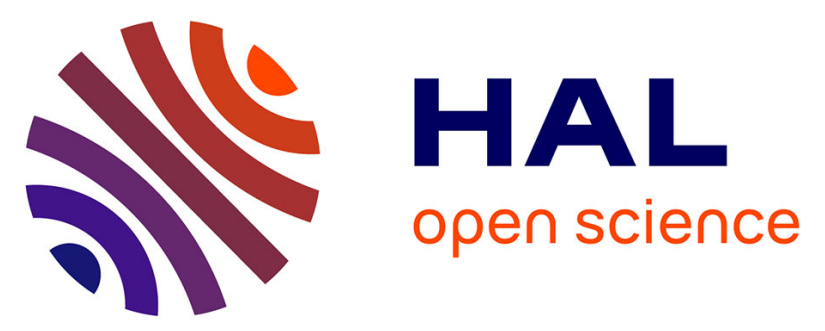

\title{
A Lumped Parameter Model to Study Atrioventricular Valve Regurgitation in Stage 1 and Changes Across Stage 2 Surgery in Single Ventricle Patients
} Sanjay Pant, Chiara Corsini, Catriona Baker, Tain-Yen Hsia, Giancarlo Pennati, Irene Vignon-Clementel

\section{To cite this version:}

Sanjay Pant, Chiara Corsini, Catriona Baker, Tain-Yen Hsia, Giancarlo Pennati, et al.. A Lumped Parameter Model to Study Atrioventricular Valve Regurgitation in Stage 1 and Changes Across Stage 2 Surgery in Single Ventricle Patients. IEEE Transactions on Biomedical Engineering, 2018, 65 (11), pp.2450-2458. 10.1109/tbme.2018.2797999 . hal-01954784

\author{
HAL Id: hal-01954784 \\ https://hal.inria.fr/hal-01954784
}

Submitted on 13 Dec 2018

HAL is a multi-disciplinary open access archive for the deposit and dissemination of scientific research documents, whether they are published or not. The documents may come from teaching and research institutions in France or abroad, or from public or private research centers.
L'archive ouverte pluridisciplinaire HAL, est destinée au dépôt et à la diffusion de documents scientifiques de niveau recherche, publiés ou non, émanant des établissements d'enseignement et de recherche français ou étrangers, des laboratoires publics ou privés. 


\title{
A Lumped Parameter Model to Study Atrioventricular Valve Regurgitation in Stage 1 and Changes Across Stage 2 Surgery in Single Ventricle Patients
}

\author{
Sanjay Pant ${ }^{\circledR}$, Chiara Corsini ${ }^{\circledR}$, Catriona Baker, Tain-Yen Hsia, Giancarlo Pennati, \\ and Irene E. Vignon-Clementel ${ }^{\mathbb{1}}$
}

\begin{abstract}
Goal: This manuscript evaluates atrioventricular valve regurgitation (AVVR) in babies born with an already very challenging heart condition, i.e., with single ventricle physiology. Although the second surgery that single ventricle patients undergo is thought to decrease AVVR, there is much controversy in the clinical literature about AVVR treatment. Methods: The effect of AVVR on Stage 1 haemodynamics and resulting acute changes from conversion to Stage 2 circulation in single ventricle patients are analyzed through lumped parameter models. Several degrees of AVVR severity are analyzed, for two types of valve regurgitation: incomplete leaflet closure and valve prolapse. Results: The models show that increasing AVVR in Stage 1 induces the following effects: first, higher stroke volume and associated decrease in ventricular end-systolic volume; second, increase in atrial volumes with V-loop enlargement in pressure-volume curves; third, pulmonary venous hypertension. The Stage 2 surgery results in volume unloading of the ventricle, thereby, driving a decrease in AVVR. However, this effect is offset by an increase in ventricular pressures resulting in a net increase in regurgitation fraction (RF) of approximately 0.1 (for example, in severe AVVR, the preoperative RF increases from $\sim 60 \%$ to $\sim 70 \%$ postoperatively). Moreover, despite some improvements to sarcomere function early after Stage 2 surgery, it may deteriorate in cases of severe AVVR. Conclusion: In patients with moderate to severe AVVR, restoration of atrioventricular valve competence prior to, or at the time of, Stage 2
\end{abstract}

Manuscript received September 13, 2017; revised December 11, 2017; accepted January 20, 2018. Date of publication January 25, 2018; date of current version October 18, 2018. This work was supported by the Leducq Foundation as a part of the Transatlantic Network of Excellence for "Multi-scale modeling of single ventricle hearts for clinical decision support" and a British Heart Foundation Clinical Research Fellowship FS/12/35/29566. (Corresponding author: Irene E. Vignon-Clementel.)

S. Pant is with the Zienkiewicz Centre for Computational Engineering, Swansea University.

C. Corsini and G. Pennati are with the Laboratory of Biological Structure Mechanics, Department of Chemistry, Materials and Chemical Engineering "Giulio Natta," Politecnico di Milano.

C. Baker and T.-Y. Hsia are with the Cardiac Unit, UCL Institute of Cardiovascular Science, and also with the Great Ormond Street Hospital for Children.

I. E. Vignon-Clementel is with the Inria Paris \& Sorbonne Universités UPMC Paris 6, Laboratoire Jacques-Louis Lions, Paris 75005, France (e-mail: irene.vignon-clementel@inria.fr).

This paper has supplementary downloadable material available at http://ieeexplore.ieee.org.

Digital Object Identifier 10.1109/TBME.2018.2797999 surgery would likely lead to improved haemodynamics and clinical outcome as the models suggest that uncorrected AVVR can worsen across Stage 2 surgery. This was found to be independent of the AVVR degree and mechanisms.

Index Terms-Atrioventricular valve, valve regurgitation, lumped parameter model, cardiovascular flow, hypoplastic left heart syndrome, incomplete leaflet closure, prolapse.

\section{INTRODUCTION}

INGLE-VENTRICLE heart defect, a condition where one $\checkmark$ of the ventricles is severely underdeveloped, affects approximately 2 in 10,000 live births. This congenital heart defect requires specialised management, including three complex open-heart surgeries to ensure a long-term meaningful life. The first surgery is done when the baby is a few-days-old, the second at around 6-months of age, and the third at 2-3 years of age. The goal of these surgeries is to achieve a series connection between the systemic and pulmonary circulations so that deoxygenated blood can flow passively into the pulmonary arteries without the need of a second ventricle. In the first surgery, Stage 1, a systemic-to-pulmonary shunt (between the aorta and pulmonary artery) is created so that the only functioning ventricle, the single ventricle, pumps blood to both systemic and pulmonary circulations in parallel. In the second surgery, Stage 2, the shunt from Stage 1 is removed and the superior vena cava (bringing deoxygenated blood from the upper body) is connected directly to the pulmonary artery. Finally, in the third stage, Stage 3, the inferior vena cava (bringing blood from the lower body) is directly connected to the pulmonary artery, thereby resulting in a series connection between the systemic and pulmonary circulations.

Significant atrioventricular valve regurgitation is known to lead to poor hemodynamics and is a major risk factor for patients with single ventricle physiology. Yamagishi and colleagues [1] reported an AVVR incidence of $68 \%$ among single ventricle patients at Stage 2 reconstruction, and other two groups found AVVR in $42 \%$ and $34 \%$, respectively, of large cohorts of neonates undergoing Stage 2 surgery with superior cavopulmonary connection (SCPC) [2], [3]. The cardiopulmonary arrangement after a Stage 1 procedure, where pulmonary blood flow is derived from a systemic-to-pulmonary shunt, results in a 
parallel circulation where both the systemic and pulmonary circulations are driven by the single ventricle. The single ventricle is thus volume overloaded, although the afterload is reduced due to the parallel circulatory arrangement. Following the Stage 2 operation, where the systemic-pulmonary arterial shunt is taken down and pulmonary blood flow is established by a superior cavopulmonary connection, the work of providing pulmonary blood flow is removed from the single ventricle (volume offloading), which can promote beneficial ventricular remodeling [2]. Paediatric cardiac surgeons have long recognized the negative impact AVVR has on patient outcome. However, as atrioventricular valve repair is technically challenging and results unpredictable, some centers would refer patients with severe AVVR for heart transplantation, while others would hope for improved atrioventricular valve function following volume offloading following Stage 2 operation. The indication for and the surgical outcomes following interstage or concomitant (at time of Stage 2 surgery) atrioventricular valve repair remains a major clinical challenge. Nonetheless, the exact detrimental effects of AVVR on both haemodynamic and ventricular performance in both Stage 1 and Stage 2 circulations remain poorly defined.

Among single ventricle patients undergoing staged palliation, higher prevalence of AVVR was observed in those with a hypoplastic left ventricle [1], [2], [4], [5], where the single ventricle is morphologically right ventricle, which functions normally in the low-pressured pulmonary circulation. Unlike the mitral valve, a tricuspid valve (or common atrioventricular valve in an atrioventricular septal defect) normally face a right ventricular systolic pressure that is typically $1 / 3$ to $1 / 4$ of the left ventricle. Therefore, not only is the right ventricle required to work as a systemic (left) ventricle, the atrioventricular valve in a patient with hypoplastic left ventricle is subjected to pressure and flow environment that it has neither the structure for nor the design to perform in. Structural valve abnormalities were identified as the primary mechanisms of regurgitation: they included dysplastic leaflets, prolapse, restricted movement of one or more leaflets and central malcoaptation, due to annular dilatation [1], [4], [6]. AVVR was routinely assessed before SCPC using echocardiography based on the ratio of the colour Doppler regurgitant jet area to the area of the atrium. AVVR was usually scored from 1 to 4 (higher score implying higher AVVR), or alternatively graded as mild if the percentage ratio was lower than $30 \%$, moderate if it was between $30 \%$ and $50 \%$, and severe if it exceeded 50\% [3]. In vivo studies have reported higher percentages of mild or moderate degrees of regurgitation, rather than severe, in single ventricle patients affected by AVVR. The criterion used to decide whether to treat or not to treat AVVR at Stage 2 surgery remain controversial. In the past, AVVR repair was reserved for the most severe manifestation or when there is significant haemodynamic compromise [3]. However, with the recognition of the importance of achieving atrioventricular valve competence in single ventricle patients, many centers have now adopted an aggressive strategy for interstage or concomitant AVV repair for AVVR less than severe, while others tend to be more conservative and reserve repair for only the moderate to severe cases [1], [4]. For example, the cases reviewed by Honjo and colleagues [4] repaired all cases of moderate AVVR and reserved repair in mild to moderate AVVR only when the valve was structurally abnormal. However, the rationale of treating mild or moderate AVVR at the cost of prolonged operation and myocardial insult remains controversial. Nonetheless, in early followup, most of the AVVR corrections concomitant with SCPC exhibited improvement in atrioventricular valve competence [1], [2], [4]. On the contrary, the majority of patients with mild or moderate pre-operative AVVR, who were not subjected to concomitant valve repair, seemed to maintain their AVVR severity after SCPC [4].

Overall, the management of AVVR in single ventricle patients is challenging as it depends not only on the valve morphology but also on the mechanical behaviour of the atrium and the ventricle, as well as on the cardiac preload and afterload conditions. Also, as there is no valve replacement therapy available, surgical repair of regurgitant atrioventricular valve during infancy has been challenging. Therefore, there has been a historical, but unproven, concept that the volume offloading provide by Stage 2 circulation can lead to reduction in the severity of AVVR and consequently delay or eliminate the need for interstage or concomitant atrioventricular valve repair. While in theory, transforming a parallel Stage 1 to an in-series Stage 2 circulation has the potential to alter the geometry and function of the atrioventricular junction, clinical experience has not been uniformly favourable. Accordingly, the present study aims to 1) quantitatively examine of the effects of varying degree of AVVR on haemodynamic and ventricular performance of the Stage 1 circulation, and 2) assess the influence of conversion to a Stage 2 SCPC circulation on acute AVVR severity. To our knowledge, this represents the very first attempt to mathematically simulate and systematically assess $\mathrm{AV}$ valve regurgitation single ventricle physiology. This work is based on a validated lumped parameter model (LPM), often referred as electric analog or zero-D model [7], [8], of the single ventricle circulation that allows for AVVR and surgical stages modelling. The methods detail the model components and the necessary changes for this study compared to previous work. The results are compared to the clinical literature, while others give insights into non-easily measurable quantities.

\section{Materials and Methods}

A lumped parameter model (LPM) abstracts the complex circulation into lumped elements that capture essential dynamical relationships between pressures and flow-rates in the heart and connected systemic and pulmonary circulations. An electrical analogy is typically used where pressure is analogous to voltage and flow-rate is analogous to electric current, resulting in a nonlinear system of ordinary differential equations. A major advantage of LPMs is that full-body closed-loop circulation can be dynamically represented over time while keeping computational costs manageable. For evaluating the effects of AVVR, representation of a closed-loop circulation is critical as ventricular loadings and heart function need to be assessed, thereby making LPM an excellent model choice. Fig. 1 shows the lumped parameter models for Stage 1 and Stage 2 single-ventricle physiology. Note that in Stage 1, a systemic-to-pulmonary shunt (SH) 


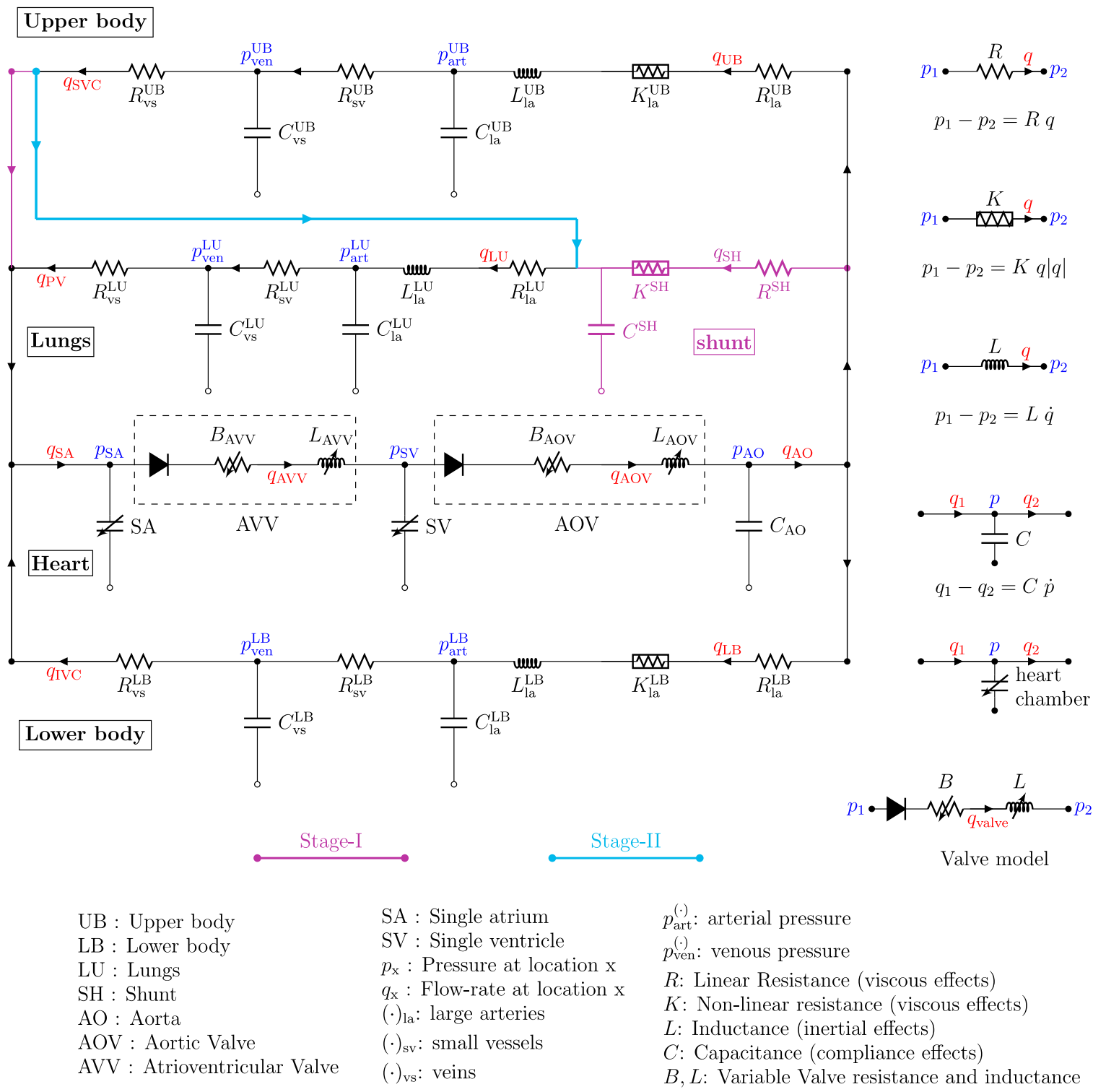

Fig. 1. Lumped parameter model for Stage 1 and Stage 2 single-ventricle physiology: pressures at major locations are represented in blue and flow-rates in red (see key for details).

connects the aorta (AO) to the pulmonary artery (PA) which is removed in Stage 2 surgery when the superior vena cava (SVC) is connected to the PA. In the LPM, the viscous losses are modelled by linear $(R)$ and non-linear $(K)$ resistances, the compliance of the large arteries and veins is modelled by capacitances $(C)$, and blood inertia is modelled by inductances $(L)$. The heart chambers are depicted as variable capacitances and the valves are depicted as a combination of diodes and time-varying resistances $(B)$ and inductances $(L)$. The heart models with the addition of passive viscosity and the valve models to simulate regurgitation are described below.

\section{A. Heart Model}

A one-fibre model [8]-[11] is used to describe the two heart chambers (single ventricle and single atrium) in single ventricle physiology. In this model, the relationship between pressure in the chamber $p$, volume of the chamber $V$, fibre stress $\sigma_{\mathrm{f}}$, and the myocardial wall volume $V_{\mathrm{w}}$ is described as $\sigma_{\mathrm{f}} / p=\left(1+3 V / V_{\mathrm{w}}\right)$. The active stress depends on sarcomere (the functional unit of the fibre) length $(l)$, sarcomere shortening velocity $\left(v_{s}\right)$, and time elapsed since activation $\left(t_{a}\right)$ as follows

$$
\sigma_{\mathrm{a}}=T_{\mathrm{a} 0} f(l) g\left(t_{\mathrm{a}}\right) h\left(v_{\mathrm{s}}\right)
$$

where $T_{\mathrm{a} 0}$ is a material constant, $f(l)$ represents the sarcomere force-length relationship, $g\left(t_{a}\right)$ models the contraction of the chamber, $h\left(v_{s}\right)$ models sarcomere active viscosity. These dependences are described in earlier works by the authors [8], [11] and are unmodified in this study. On the contrary, some changes are here introduced to better model the passive behaviour of the myocardium. Namely, while in the previous model the passive stress depended only on the sarcomere length, the effect of the passive myocardium viscosity is additionally introduced in this 
study. This modification is necessary in order to have realistic pressure-volume (PV) loops of the atrium. Indeed, in the absence of passive viscosity in the sarcomere model, the peculiar figureof-eight of the atrial PV plot, with two distinctive loops (A-loop and V-loop [12], [13]) cannot be reproduced by the model (the V-loop is absent). The capability of the model to properly mimic the atrial PV loops is important for the current study, since the AVVR model will be verified by comparison with some animal experiments, where A-loops and V-loops were analyzed before and after the creation of acute experimental tricuspid regurgitations [14]. If $V_{0}$ and $l_{0}$ describe the volume of the heart chamber and the sarcomere length, respectively, at zero transmural pressure under passive conditions, then the fibre stretch $\lambda$ at any given volume $V$ can be written as

$$
\lambda=l / l_{0}=\left[\left(1+\left(3 V / V_{\mathrm{w}}\right)\right) /\left(1+\left(3 V_{0} / V_{\mathrm{w}}\right)\right)\right]^{1 / 3} .
$$

The passive stress is then expressed as follows

$$
\sigma_{\mathrm{p}}=\left\{\begin{array}{cl}
0, & \text { if } \lambda<1 \\
T_{\mathrm{p} 0}\left(e^{\left\{c_{\mathrm{p}}(\lambda-1)\right\}}-1\right)(1+\zeta(\dot{\lambda})), & \text { if } \lambda \geq 1
\end{array}\right. \text { ， }
$$

where $T_{\mathrm{p} 0}$ and $c_{\mathrm{p}}$ are sarcomere material constants, and compared to the previous works [8]-[11] the additional term to model viscous effects is $(1+\zeta(\dot{\lambda}))$ with $\dot{\lambda}=d \lambda / d t$ and $\zeta(\dot{\lambda})$ given as

$$
\zeta(\dot{\lambda})=\mu \frac{|\dot{\lambda}|}{\dot{\lambda}}(|\dot{\lambda}|)^{\phi}=\operatorname{sgn}(\dot{\lambda}) \mu(|\dot{\lambda}|)^{\phi}
$$

where $\mu$ and $\phi$ are material constants, and $\operatorname{sgn}(x)$ denotes the sign function: $\operatorname{sgn}(x)=-1$ if $x<0$, and $\operatorname{sgn}(x)=+1$ if $x>$ 0 . Based on the experimental results presented in [15], [16], the multiplication of the new term $(1+\zeta(\dot{\lambda}))$ in (3) is chosen to ensure zero passive stress in an unstretched fibre: as the stretch goes to one, i.e. $\lambda \rightarrow 1$, the total passive stress will vanish as $\left(\exp \left\{c_{\mathrm{p}}(\lambda-1)\right\}-1\right) \rightarrow 0$. The dependence of $\zeta(\dot{\lambda})$ on the time derivative of fibre stretch, with $\phi=0.275$, is used to describe the influence of the rate of shortening/lengthening of the fibre observed in large animals [15]. Moreover, a value of $\mu=0.3$ (units are $s^{0.275}$ ) is set to obtain realistic human atrial PV loops [12], [13].

\section{B. Valve Description and Regurgitation Model}

The valve model is adopted from previous studies [8], [11] to model regurgitation due to incomplete leaflet closure and prolapse. The pressure drop $\Delta p$ across a valve is described as a function of flow-rate $q$ as follows

$$
\Delta p=B q|q|+L \frac{d q}{d t}, \quad B=\frac{\rho}{2 A_{\mathrm{eff}}^{2}}, \quad L=\frac{\rho l_{\mathrm{eff}}}{A_{\mathrm{eff}}},
$$

where $\rho$ is the density of blood, and $A_{\text {eff }}$ and $l_{\text {eff }}$ denote the effective opening area and effective length of the valve, respectively. The dynamically changing valve area $A_{\text {eff }}(t)$ is described by a variable $\xi(t)$ as follows

$$
\begin{aligned}
& A_{\text {eff }}(t)=
\end{aligned}
$$

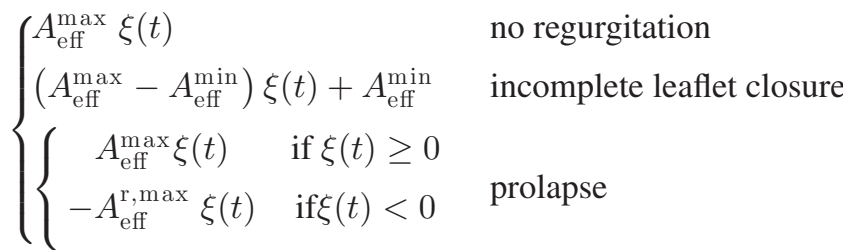

where $-1 \leq \xi \leq 1$ and the parameters $A_{\mathrm{eff}}^{\max }, A_{\mathrm{eff}}^{\min }$, and $A_{\mathrm{eff}}^{\mathrm{r}, \max }$, represent the maximum effective area, minimum effective area, and the maximum regurgitant area under prolapse, respectively. Note that $\xi<0$ only for prolapse. The following ordinary differential equations describe the dependence of the time derivative of $\xi$ on the pressure difference $\Delta p$ across the valve (see [8] for details)

$$
\begin{aligned}
& \dot{\xi}= \\
& \begin{cases}(1-\xi) K_{\mathrm{vo}} \Delta p & \text { if } \Delta p \geq 0 \\
\xi K_{\mathrm{vc}} \Delta p & \text { if } \Delta p_{\mathrm{rg}} \leq \Delta p<0 \& \xi \geq 0 \\
(1+\xi) K_{\mathrm{vo}}^{\mathrm{r}}\left(\Delta p-\Delta p_{\mathrm{rg}}\right) & \text { if } \Delta p \leq \Delta p_{\mathrm{rg}} \\
-\xi K_{\mathrm{vc}}^{\mathrm{r}}\left(\Delta p-\Delta p_{\mathrm{rg}}\right) & \text { if } \Delta p>\Delta p_{\mathrm{rg}} \& \xi<0\end{cases}
\end{aligned}
$$

where $\Delta p_{\text {rg }}$ is the threshold pressure gradient beyond which prolapse of the valve begins, and $K_{(\cdot)}$ are proportionality parameters.

Atrioventricular valve regurgitation is quantified by the regurgitant volume flow-rate (RVF, also referred just as 'regurgitant volume' in clinical literature) and the regurgitation fraction (RF) defined as follows

$$
\begin{aligned}
\mathrm{RVF} & =\mathrm{CO}-\bar{q}_{\mathrm{AO}}, \quad \text { and } \\
\mathrm{RF} & =\mathrm{RVF} / \mathrm{CO}, \quad \text { with } \\
\mathrm{CO} & =\frac{\mathrm{EDV}-\mathrm{ESV}}{T_{c}},
\end{aligned}
$$

where $T_{c}$ denotes one cardiac cycle, $\mathrm{CO}$ represents the cardiac output measured through the difference between the enddiastolic (EDV) and end-systolic volumes (ESV) of the single ventricle, and $\bar{q}_{\mathrm{AO}}$ represents the average flow through the aorta in one cardiac cycle, i.e. $\bar{q}_{\mathrm{AO}}=\frac{1}{T_{c}} \int_{0}^{T_{c}} q_{\mathrm{AO}}(t) d t$, where $q_{\mathrm{AO}}(t)$ represents the instantaneous flow-rate through the aorta (see Fig. 1).

\section{Simulations Performed}

First, in Stage 1 configuration (pre-operative), the effect of an increasing degree of AVVR is simulated. In case of incomplete leaflet closure, AVVR is modelled by successively increasing the minimum effective area of the valve $A_{\text {eff }}^{\min }$ from zero (when there is no regurgitation) to achieve values of approximately 0.2 , 0.4 , and 0.6 for the regurgitation fractions (RFs) representing mild, moderate, and severe regurgitation [3], respectively. Similarly, regurgitation due to prolapse is modelled by successively 
TABLE I

EFFeCt OF StAGE 1 to Stage 2 SURgery at VARYing LEVELS OF PRE-Operative Regurgitation Fractions (RF)

\begin{tabular}{|c|c|c|c|c|c|c|c|}
\hline \multicolumn{8}{|c|}{ Incomplete leaflet closure } \\
\hline \multicolumn{2}{|l|}{ AVVR } & \multicolumn{3}{|c|}{ pre-operative } & \multicolumn{3}{|c|}{ post-operative } \\
\hline & $\begin{array}{l}A_{\mathrm{eff}^{\mathrm{min}}} \\
\left(\mathrm{cm}^{2}\right)\end{array}$ & $\begin{array}{l}\mathrm{CO} \\
(\mathrm{ml} / \mathrm{s})\end{array}$ & $\begin{array}{l}\mathrm{RVF} \\
(\mathrm{ml} / \mathrm{s})\end{array}$ & $\mathrm{RF}$ & $\begin{array}{l}\mathrm{CO} \\
(\mathrm{ml} / \mathrm{s})\end{array}$ & $\begin{array}{l}\text { RVF } \\
(\mathrm{ml} / \mathrm{s})\end{array}$ & $\mathrm{RF}$ \\
\hline none & 0.000 & 25.00 & 00.00 & 0.00 & 18.68 & 00.00 & 0.00 \\
\hline mild & 0.032 & 28.15 & 05.71 & 0.20 & 23.02 & 06.38 & 0.28 \\
\hline moderate & 0.084 & 31.66 & 12.73 & 0.40 & 28.22 & 14.45 & 0.51 \\
\hline severe & 0.201 & 34.62 & 20.79 & 0.60 & 32.92 & 23.29 & 0.71 \\
\hline \multicolumn{8}{|c|}{ Valve Prolapse (threshold pressure $\Delta P_{\mathrm{rg}}=25 \mathrm{mmHg}$ ) } \\
\hline \multirow[t]{2}{*}{ AVVR } & & \multicolumn{3}{|c|}{ pre-operative } & \multicolumn{3}{|c|}{ post-operative } \\
\hline & $A_{\mathrm{eff}}^{\mathrm{r}, \max }$ & $\mathrm{CO}$ & RVF & $\mathrm{RF}$ & $\mathrm{CO}$ & RVF & $\overline{\mathrm{RF}}$ \\
\hline none & 0.000 & 25.00 & 00.00 & 0.00 & 18.68 & 00.00 & 0.00 \\
\hline mild & 0.036 & 27.93 & 05.64 & 0.20 & 23.01 & 06.52 & 0.28 \\
\hline moderate & 0.104 & 30.58 & 12.34 & 0.40 & 27.84 & 14.66 & 0.52 \\
\hline severe & 0.332 & 32.24 & 19.36 & 0.60 & 30.47 & 22.47 & 0.73 \\
\hline
\end{tabular}
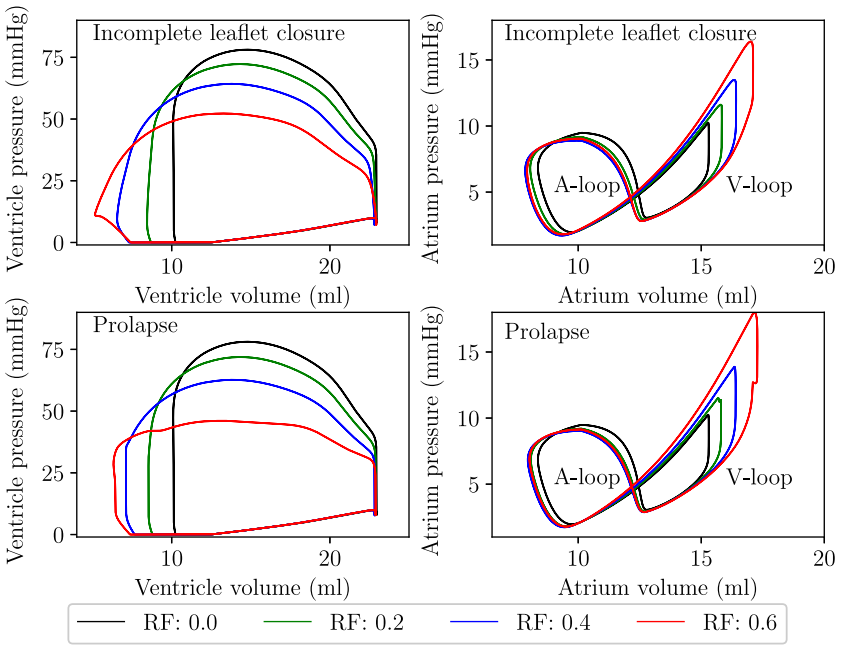

Fig. 2. Stage 1: Effect of increasing regurgitation fraction (RF) due to incomplete leaflet closure and valve prolapse $\left(\Delta p_{\mathrm{rg}}=25 \mathrm{mmHg}\right)$ on atrial and ventricular PV loops. Note: the effect of $\Delta p_{\text {rg }}$ on PV loops is presented in appendix.

increasing $A_{\text {eff }}^{\mathrm{r}, \mathrm{max}}$ in Stage 1 to achieve the same three levels of RFs. All the prolapse cases were investigated for three different values of $\Delta p_{\text {rg }}(10,25$, and $35 \mathrm{mmHg})$. As a second step, postoperative (Stage 2) simulations are run with the corresponding values of $A_{\mathrm{eff}}^{\mathrm{min}}$ and $A_{\mathrm{eff}}^{\mathrm{r}, \mathrm{max}}$ to study how RVFs and RFs change as a result of the conversion to a SCPC circulation model.

Except for the change of circulation topology from Stage 1 to Stage 2 (Fig. 1) and the AVVR model sub-part, the same model parameters are used for all the simulations. These circulatory parameters (shown in Fig. 1) have been identified in our previous work [8], [11] from a 3 month old patient with a body surface area of $0.26 \mathrm{~m}^{2}$, who has a hypoplastic right ventricle due to tricuspid and pulmonary atresia (see [8, Table 3] for patient-A; tables also available as supplementary material to this article). The patient-specific parameter identification was performed through the unscented Kalman Filter (UKF), a recursive Bayesian method. Clinical measurements, i.e. the dynamic variation over the cardiac cycle of the aortic and atrial pressures, systemic flow-rates (aortic, pulmonary, upper body, and lower body), and venous flow-rates (pulmonary, inferior vena cava, and superior vena cava) were used as UKF observables (targets). Starting from initial guesses of the model parameters, the UKF provides a parameter evolution strategy such that the measurements are reproduced by the model outputs. The UKF parameter estimates were validated quantitatively by comparing model output with the measured ventricular pressure, and qualitatively by comparing valvular flow-rates with measured Doppler flow-rates.

For AVVR modelling in this manuscript, the maximum annulus area is set to $A_{\mathrm{eff}}^{\mathrm{min}}=1.10 \mathrm{~cm}^{2}$, and the values of $A_{\mathrm{eff}}^{\mathrm{min}}$ and $A_{\text {eff }}^{\mathrm{r}, \max }$ for incomplete leaflet closure and valve prolapse, respectively, are shown in Table I for various degrees of AVVR assessed.

\section{Results And Discussion}

\section{A. Effect of AVVR on Heart Function (Ventricle and Atrial PV Loops)}

Fig. 2 depicts the ventricular and atrial PV loops in Stage 1, for increasing acute AVVR and the two mechanisms of regurgitation (incomplete leaflet closure or prolapse). For valve prolapse, only the results for $\Delta p_{\text {rg }}$ of $25 \mathrm{mmHg}$ are presented in this study since simulations for all the three $\Delta p_{\text {rg }}$ values provided similar conclusions with respect to AVVR effects (results for all the three cases are presented in appendix). For the case without regurgitation $(\mathrm{RF}=0)$, the ventricular PV loop has the two classical isovolumic phases and the atrial PV loop shows the figure-of-eight, with two loops of similar area. However, as regurgitation is successively increased (by increasing the regurgitant area $A_{\mathrm{eff}}^{\mathrm{min}}$ or $A_{\mathrm{eff}}^{\mathrm{r}, \mathrm{max}}$ ), the isovolumic phases of ventricular PV loops clearly disappear in case of incomplete leaflet closure while are still present for prolapse. Furthermore, the following changes in the PV loops of the single ventricle are observed irrespective of the mechanism (incomplete leaflet closure or prolapse) for regurgitation: i) the end diastolic volume remains largely unchanged (an insignificant increase is observed); ii) the end systolic volume decreases; iii) with corresponding higher stroke volume; and iv) the peak systolic pressure reduces significantly. In PV loops of the single atrium, two typically observed loops making a figure-of-eight configuration are seen. These loops correspond to the A-wave and the $\mathrm{V}$-wave observed in right atrial pressure recordings. Note that the presence of $\mathrm{V}$ loop in the model results is a direct consequence of the addition of viscous effects in the model; this loop is absent if viscous effects are ignored as in our previous studies [8], [11]. In the atrial PV loops, as regurgitation fraction is increased: i) the overall area of the PV loop (corresponding to atrium power) increases, driven by an increase in maximum atrial volume (the volume at which the AV valve opens); ii) the area of the A-loop does not change remarkably (it increases marginally from no regurgitation to mild regurgitation and thereafter, on further increasing regurgitation, remains approximately unchanged) 
while iii) the area of the V-loop notably increases with increasing regurgitation.

\section{B. Discussion of AVVR Effect on PV Loops in Light of the Literature}

The model predictions about the changes occurring in PV loops of both ventricle and atrium when the regurgitation severity increases are consistent with the reported response to acute AVVR, as deduced from animal experiments. Gaasch \& Meyer [17] reported that the ventricular response to acute AVVR is reduced afterload, increased ejection fraction, and a higher stroke volume. This is precisely what is observed in the model results (see Fig. 2). They also suggested that normal ventricular compliance limits the increase in end diastolic volume. In the model results, the end diastolic volume remains largely unchanged (an insignificant increase is numerically observed). Harpole et al. [18] studied ventricular PV loop changes in patients undergoing mitral valve replacement and reported that patients with mitral valve regurgitation demonstrated a decrease in ejection fraction and an increase in end systolic pressures after valve replacement. Since the onset of acute AVVR is the opposite of a regurgitant mitral valve replacement surgery, the model results show a reversed trend. They also reported that changes in end diastolic data (pressure and volume) were not different pre- and post-surgery, consistent with the model results. Lastly, Gaemperli et al. [19] recorded ventricular PV loops in patients with mitral valve regurgitation undergoing percutaneous mitral valve repair. Consistent with the reversed trend observed in model results, they reported that in majority of the patients the end systolic pressure increases and end systolic volume increases. Similar to Harpole et al. [18], they also reported that acutely, the end diastolic volume does not change (their data showed a $6 \%$ decrease post mitral valve repair with a p-value of 0.18), but a 1-8 month follow-up demonstrates a step-wise reduction in end diastolic volumes.

Regarding the atrial PV loops, the observed monotone increase of V-loop size with increasing AVVR severity agrees well with the data reported by Miller et al. [14] in pig experiments. Overall, the inclusion of viscous effects in the passive behaviour of the sarcomere coupled with the proposed singleventricle LPM show a good agreement with animal observations with increasing acute AVVR.

\section{AVVR Effect on Hemodynamics and Differences Between the 2 Regurgitation Types}

Fig. 3 (also see Fig. 1 for pressure and flow locations) shows how the major haemodynamics parameters change with increasing acute AVVR. Consistent with a decreasing peak systolic pressure in the single ventricle, the aortic pressure $p_{\mathrm{AO}}$, and hence the aortic flow-rate $q_{\mathrm{AO}}$, decrease with increasing RF. As expected, $q_{\mathrm{AVV}}$ shows a larger negative flow-rate as $\mathrm{RF}$ increases. However, differences in retrograde flow patterns between AVVR mechanisms of incomplete leaflet closure and prolapse are observed: while for incomplete leaflet closure retrograde flow occurs during entire systole, for prolapse the period
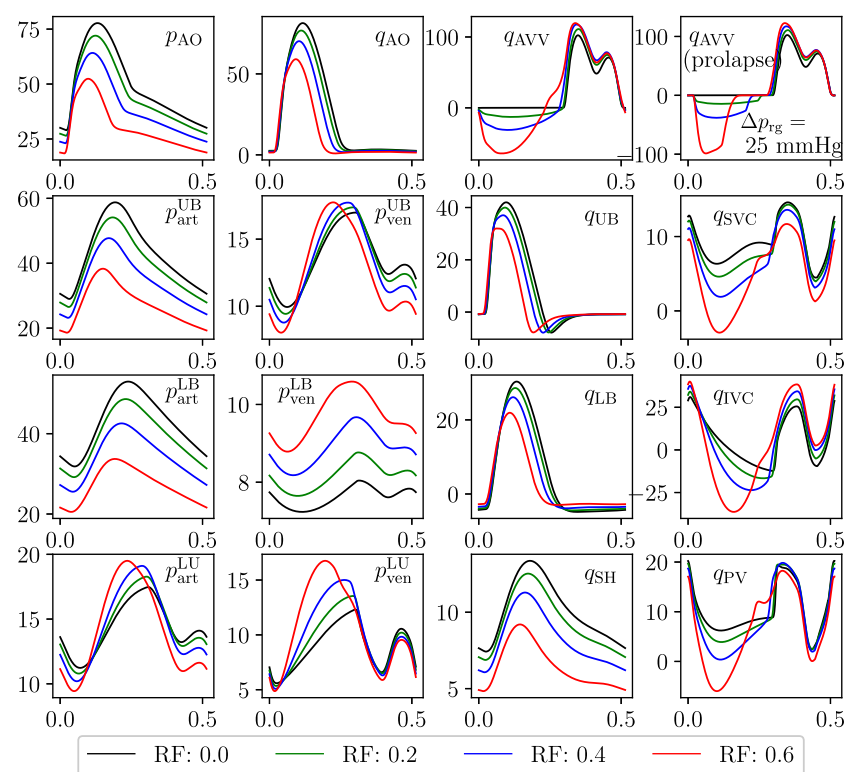

Fig. 3. Stage 1: Effect of increasing regurgitation fraction on global haemodynamics. All the plots are for incomplete leaflet closure, except the top-right plot which shows atrioventricular flow-rate for valve prolapse. Pressures are in $\mathrm{mmHg}$ and flow-rates are in $\mathrm{ml} / \mathrm{s}$.

of retrograde flow-rate is restricted due to $\Delta p_{\mathrm{rg}}$ and is skewed towards early systole. This explains the different model results, in terms of the ventricular PV loops, between AVVR due to incomplete leaflet closure and prolapse. Indeed, since incomplete leaflet closure leads to persistent non-zero opening area for the valve, isovolumic contraction and relaxation are absent; on the contrary, when AVVR is due to prolapse, isovolumic phases are present until $\Delta p \leq \Delta p_{\mathrm{rg}}$, since in these phases valve regurgitation does not occur.

The plot of $q_{\mathrm{AO}}$ shows that the aortic valve ejection time decreases with increasing AVVR. This decrease in the opening duration of the aortic valve may be used as a surrogate to monitor AVVR in Stage 1 patients. For the lower body (LB) and upper body (UB) districts, the arterial pressures $p_{\text {art }}^{(\cdot)}$ show a consistent decrease in average pressures and insignificant changes in the venous pressures. However, for the lung (LU) district, the venous pressure $p_{\text {ven }}^{\mathrm{LU}}$ shows an almost $50 \%$ increase in peak values. This suggests that pulmonary venous hypertension is a likely effect of increasing acute AVVR. This observation is supported by clinical observations where pulmonary hypertension/congestion is frequently a sequalae of significant AVVR [17]. Lastly, the arterial flow-rates $\left(q_{\mathrm{UB}}, q_{\mathrm{LB}}\right.$, and $\left.q_{\mathrm{SH}}\right)$ show a consistent decrease with increasing AVVR while the venous flow-rates $\left(q_{\mathrm{SVC}}, q_{\mathrm{IVC}}\right.$, and $\left.q_{\mathrm{PV}}\right)$ show an increase in pulsatility (the peak flow-rates are amplified) and flow reversal, in particular in the inferior vena cava (IVC). The model results indicate that the observed modifications in the time-tracings of flows (i.e. the decrease in the ejection time of the aortic valve and the increased pulsatility of venous flows) could be clinically used to monitor AVVR progression in Stage 1 patients (e.g. through a Doppler velocity analysis), as already suggested for adults [20], [21]. 


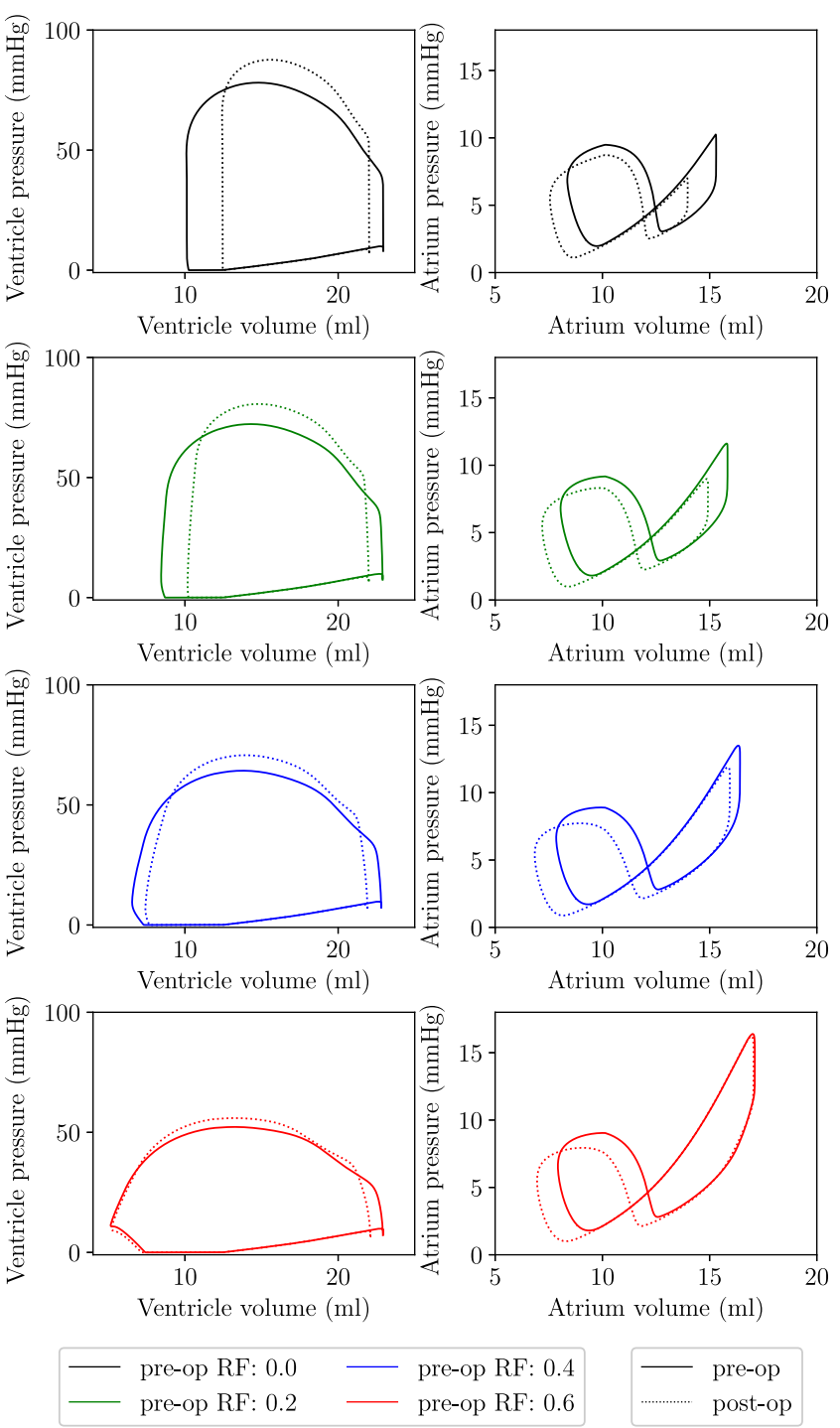

Fig. 4. Stage 1 (pre-operative) to Stage 2 (post-operative): Effect of increasing regurgitation fraction (RF) due to incomplete leaflet closure on atrial and ventricular PV loops.

\section{Effect of Conversion From Stage 1 to Stage 2 Circulation on AVVR}

Fig. 4 shows a comparison of pre-operative and post-operative PV loops for the single ventricle and single atrium for no, mild, moderate, and severe pre-operative AVVR due to incomplete leaflet closure (the behaviour for AVVR due to prolapse yields the same observations, and hence the results for prolapse are omitted). The following observations are made: i) Stage 2 surgery results in a volume unloading (decrease in end diastolic volume and increase in end systolic volume thereby resulting in a lower stroke volume) of the single ventricle; ii) the peak systolic pressure in the ventricle is increased due to Stage 2 surgery; and iii) the size of atrial A-loop remains unchanged while the V-loop shows reduction in size (the difference reducing with increasing pre-operative AVVR), except for severe AVVR where the A-loop increases in size. From Stage 1 to Stage 2 surgery, the net effect of the opposing forces-volume unloading

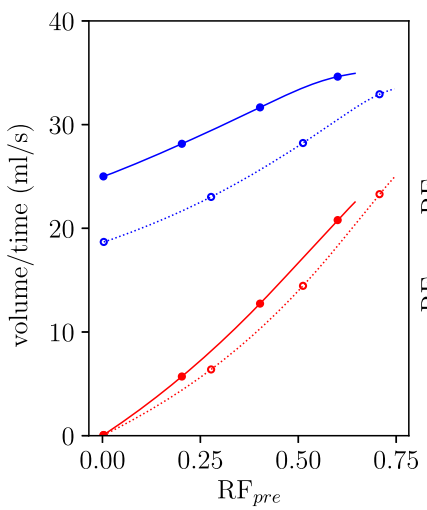

(a)

$$
\begin{array}{llll}
- & \text { pre-op CO } & - & \text { pre-op RVF } \\
\cdots \cdots \cdots \cdots \cdots \cdots & \text { post-op CO } & \cdots \cdots \cdots \cdots \cdots & \text { post-op RVF }
\end{array}
$$

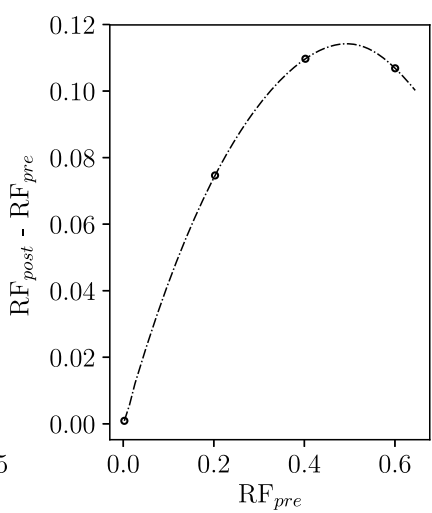

(b)
Fig. 5. Stage 1 (pre-operative) to Stage 2 (post-operative): (a) Effect of increasing pre-operative regurgitation fraction (RF) due to incomplete leaflet closure on cardiac output (CO), regurgitant volume flowrate (RVF), and (b) change in regurgitation fraction due to surgery. The markers indicate pre-operative RFs of $0.0,0.2,0.4$, and 0.6 , and the continuous lines are generated by sampling 150 values of $A_{\mathrm{eff}}^{\mathrm{min}}$ between $0.0 \mathrm{~cm}^{2}$ and $0.25 \mathrm{~cm}^{2}$.

driving a reduction in AVVR while increase in systolic pressure driving an increase in AVVR-is interesting. It is observed that despite volume unloading, the increase in systolic ventricle pressures results in a net increase in the regurgitation fraction, see Table I. This net increase in the RF is lower $(\sim 0.08)$ for mild pre-operative AVVR and higher $(\sim 0.11-0.13)$ for moderate and severe pre-operative AVVR. On average, it can be concluded that the Stage 2 surgery results in an increase in RF of approximately 0.1 . Since RF is expressed relative to the cardiac output, see (10), and the cardiac output itself changes due to Stage 2 surgery, it is important to assess the changes in cardiac output and regurgitant volume flow-rates to study AVVR. Fig. 5 depicts how CO and RVF vary both pre- and post-operatively with increasing pre-operative RF. In Stage 1, as expected for increasing RF, it is observed that both $\mathrm{CO}$ and RVF increase with increasing RF but the rise in RVF is steeper than the rise in CO. A similar trend is observed for Stage 2 haemodynamics. However, when comparing the Stage 1 to Stage 2 haemodynamics, it is observed that the drop in CO due to surgery (solid blue to dashed blue lines in Fig. 5) is significantly larger than the drop in RVF (solid red to dashed red lines), thereby resulting in an overall increase in RF.

An important advantage of the one-fibre model employed in this study is that sarcomere behaviour in terms of their operating range in the force-length relationship, $f(l)$ in (1), can be investigated. These are shown in Fig. 6: the solid grey line shows $f(l)$, i.e. the normalised contraction force generated by a sarcomere of length $l$ (determined by the volume of the chamber), while the bold and dotted lines indicate the operating regions of the sarcomeres for both pre- and post-operative physiologies, respectively, at different levels of pre-operative RFs. Ventricular sarcomere function is improved for all levels of AVVR due to ventricular unloading which leads to a leftwards shift (along the positive slope region of $f(l)-l$ curve) in peak sarcom- 

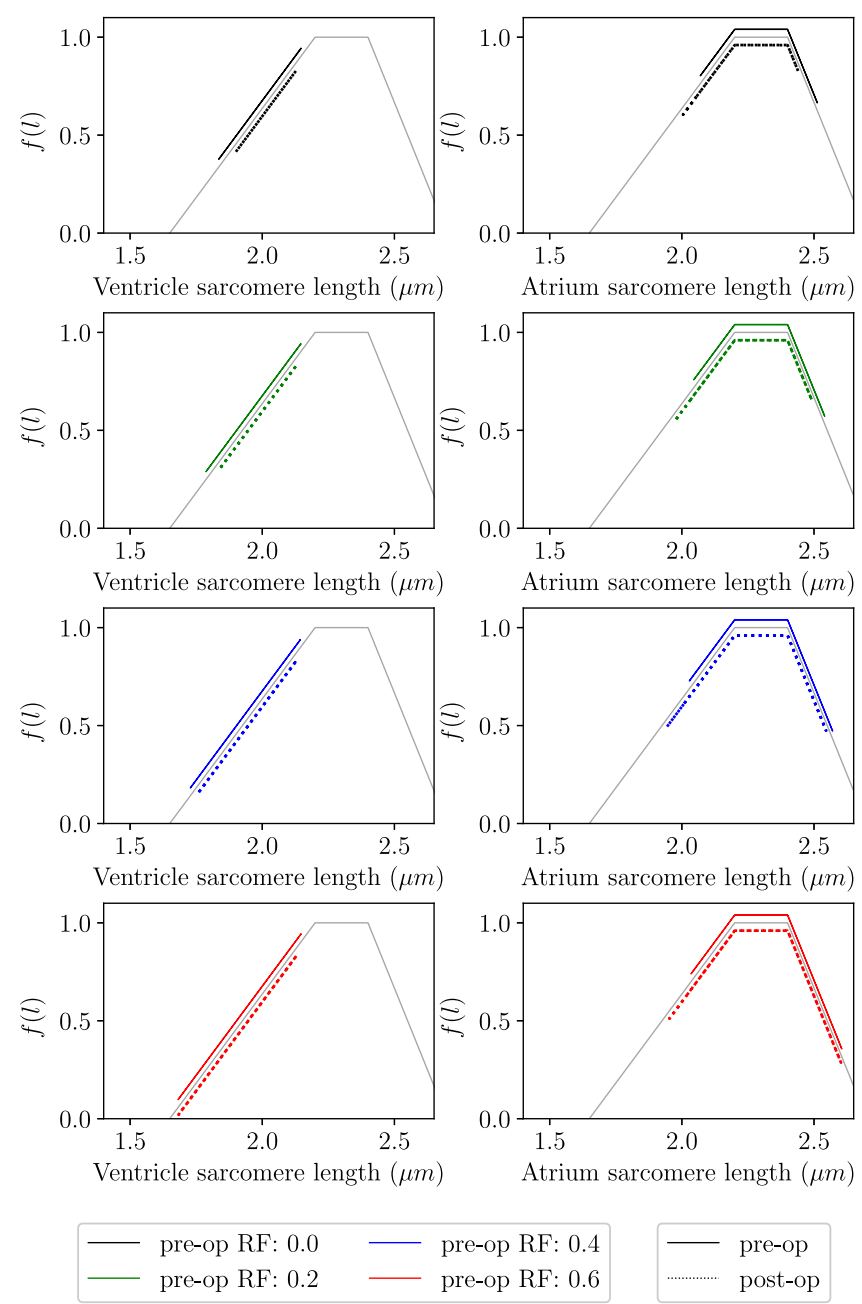

Fig. 6. Stage 1 to Stage 2 with incomplete leaflet closure: Change in the operating region of ventricular and atrial sarcomere.

ere length. This is beneficial as the ventricular sarcomeres can stretch more, if need be through an increase in end-diastolic volume, and still be able to generate a higher force of contraction. The atrial sarcomeres are observed to partly operate in the decompensated, downward slope, region of the $f(l)-l$ curve. This is likely to be a patient-specific observation and depends on the estimate of model parameters. Nevertheless, for no, mild, and moderate AVVR, an improvement (reduction in peak sarcomere length) in atrial sarcomere function is observed. This improvement decreases as AVVR levels are increased, eventually leading to deterioration of atrial sarcomere function for severe AVVR post Stage 2 surgery.

\section{E. Limitations}

The lumped parameter model adopted for the atrioventricular valve, despite showing a satisfactory behavior in describing the haemodynamics of single ventricle patients with AVVR, needs some improvements. Indeed, the maximum annulus area $A_{\text {eff }}^{\max }$ in the model, see equation (6), is assumed to remain constant between the pre- and post-operative patient states. According to clinical observations, it is likely that the heart volume unloading

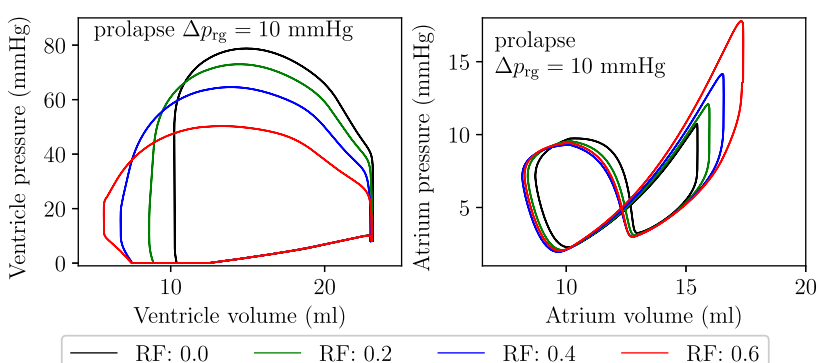

(a)

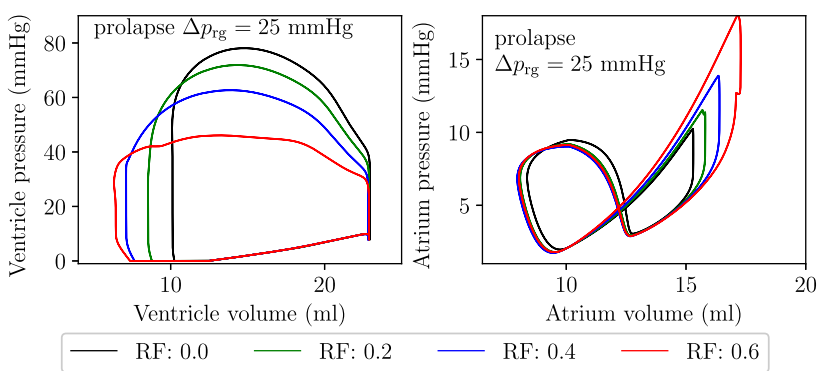

(b)
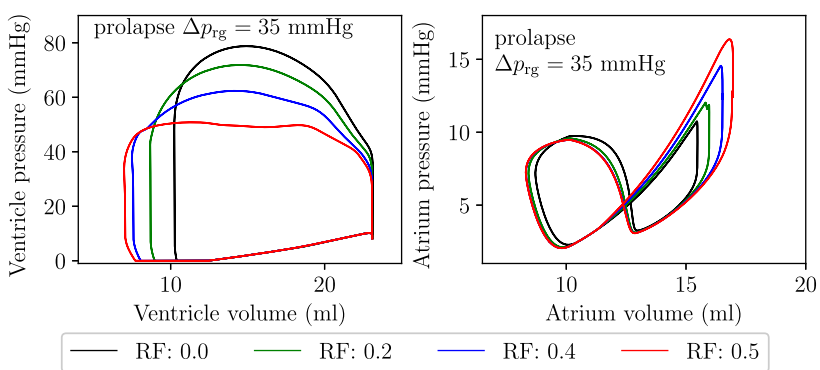

(c)

Fig. 7. Stage 1: Effect of increasing regurgitation fraction (RF) due to valve prolapse on atrial and ventricular PV loops.

due to Stage 2 surgery will result in some degree of reduction of the annulus area (which may be further affected by adaptation over the longer term) and, in turn, may affect the AVVR extent. Some groups found that the volume unloading caused by a Stage 2 procedure might have beneficial effects on AVVR without the need of treatment. Michelfelder and coworkers [6] showed reduction in the tricuspid annular dilatation rate and general improvement in valve insufficiency in all patients but one who had severe AVVR and ventricular dysfunction. Mahle and group [3] reported mid-term improvement in $22 \%$ of patients with moderate or severe pre-operative AVVR who did not undergo concomitant valve repair. Nevertheless, a much higher percentage $(67 \%)$ persisted in significant AVVR.

Furthermore, while the current study evaluated only acute changes in AVVR due to Stage 2 surgery, additional vascular mechanisms may affect the mid/long-term post-operative hemodynamics. More recently, magnetic resonance data showed that after SCPC aortopulmonary collaterals might develop so that their flow might neutralize the volume unloading effect brought by Stage 2 . This would partly explain the annular dilatation reported by Yamagishi and colleagues [1] at mid-term in patients with absent or mild pre-operative valve regurgitation who were 
not subjected to concomitant valve repair. To further increase the confidence in the model results (at least as predictions of the short-term post-operative behaviour), an advanced valve model, where the maximum annulus area is a function of the maximum (end diastolic) ventricular volume, is required. Such a model, however, requires additional clinical measurements of annulus areas at varying preloads for both model construction and validation. Furthermore, while the pre-operative model presented in this study is customised for a patient without AVVR and validated through numerous clinical measurement, it is necessary to obtain patient-specific data for a group of single ventricle patients with various AVVR degrees. Namely, together with preoperative data to build the models, post-operative AVVR measurements are demanded for further validation. These two perspectives form the primary areas of future work for this study.

\section{CONCLUSION}

The effect of increasing AVVR on Stage 1 haemodynamics and subsequent changes due to Stage 2 surgery in single ventricle physiology are analysed through a lumped parameter model. In Stage 1 circulation the following changes to haemodynamics are observed as regurgitation fraction is increased: i) insignificant increase in ventricular end diastolic volume; ii) decrease in ventricular end systolic volume; iii) increase in stroke volume; iv) mild increase in the size of the atrial A-loop; iv) significant increase in the size of atrial V-loop; and v) increase in the likelihood of pulmonary venous hypertension. Two effects of Stage 2 surgery that affect post-operative acute AVVR are identified: a) reduction in end diastolic volume and increase in end systolic volume thereby resulting in a decrease in stroke volume; and b) increase in systolic pressures. The net effect of these two opposing forces-volume unloading driving a reduction in AVVR while the increased systolic pressures driving an increase in AVVR-is a post-operative increase in regurgitation fraction of approximately 0.1 (the pre-operative RFs of $\sim 20 \%, \sim 40 \%$, and $\sim 60 \%$ change to post-operative RFs of $\sim 30 \%, \sim 50 \%$, and $\sim 70 \%$, respectively). This increase in regurgitation fraction appears to be independent of both the mechanism of AVVR (incomplete leaflet closure or valve prolapse) and the degree of AVVR (mild, moderate, or severe), and is mostly driven by the decrease in cardiac output following Stage 2 surgery. While the Stage 2 surgery, in general, results in better sarcomere function (in terms of the operating range of the sarcomere in the force-length relationship), it is demonstrated that in severe AVVR, deterioration of sarcomere function can still occur. Thus, the model results suggest that AVVR is not reduced following Stage 2 conversion as the benefit of volume unloading is offset by higher ventricular systolic pressures. Therefore, in situations where significant AVVR is present in single ventricle patients, interstage or concomitant atrioventricular valve repair would likely lead to better haemodynamics and ventricular physiology than leaving the atrioventricular valve alone.

\section{APPENDIX}

Fig. 7 shows the ventricle and atrium PV loop changes during Stage 1 as AVVR due to prolapse is increased for three different levels of $\Delta p_{\text {rg }}$. It is observed that the overall behaviour, irrespec- tive of $\Delta p_{\mathrm{rg}}$, is similar. Note that for $\Delta p_{\mathrm{rg}}$, the maximum RF achieved is 0.5 as opposed to the representative severe AVVR of 0.6 utilised in this study. Since, increasing RF results in a decrease in ventricular pressure and regurgitation due to prolapse occurs once the ventricular pressure increases beyond $\Delta p_{\text {rg }}$, it is observed that there is a limit to the highest RF achievable despite extremely high values of the regurgitant area $A_{\mathrm{eff}}^{\mathrm{r}, \max }$.

\section{ACKNOWLEDGMENT}

Conflict of Interest: There are no conflicts of interests.

\section{REFERENCES}

[1] S. Yamagishi et al., "Influence of bidirectional cavopulmonary anastomosis and concomitant valve repair on atrioventricular valve annulus and function," Ann. Thoracic Surg., vol. 98, no. 2, pp. 641-647, 2014.

[2] T. M. Lee et al., "Risk factor analysis for second-stage palliation of single ventricle anatomy," Ann. Thoracic Surg., vol. 93, no. 2, pp. 614-619, 2012.

[3] W. T. Mahle et al., "Atrioventricular valve regurgitation in patients with single ventricle: Impact of the bidirectional cavopulmonary anastomosis," Ann. Thoracic Surg., vol. 72, no. 3, pp. 831-835, 2001.

[4] O. Honjo et al., "Atrioventricular valve repair in patients with functional single-ventricle physiology: Impact of ventricular and valve function and morphology on survival and reintervention," J. Thoracic Cardiovascular Sur., vol. 142, no. 2, pp. 326-335, 2011.

[5] J. G. Kwak et al., "Early surgical correction of atrioventricular valvular regurgitation in single-ventricle patients," Ann. Thoracic Surg., vol. 90, no. 4, pp. 1320-1323, 2010.

[6] E. C. Michelfelder et al., "Effect of superior cavopulmonary anastomosis on the rate of tricuspid annulus dilation in hypoplastic left heart syndrome," Amer. J. Cardiol., vol. 89, no. 1, pp. 96-98, 2002.

[7] Y. Shi et al., "Review of zero-d and 1-d models of blood flow in the cardiovascular system," Biomed. Eng. Online, vol. 10, no. 1, 2011, Art. no. 33.

[8] S. Pant et al., "Data assimilation and modelling of patient-specific singleventricle physiology with and without valve regurgitation," J. Biomech., vol. 49, no. 11, pp. 2162-2173, 2016.

[9] T. Arts et al., "Relation between left ventricular cavity pressure and volume and systolic fiber stress and strain in the wall," Biophys. J., vol. 59, no. 1, pp. 93-102, 1991.

[10] P. Bovendeerd et al., "Dependence of intramyocardial pressure and coronary flow on ventricular loading and contractility: A model study," Ann. Biomed. Eng., vol. 34, no. 12, pp. 1833-1845, 2006.

[11] S. Pant et al., "Inverse problems in reduced order models of cardiovascular haemodynamics: aspects of data assimilation and heart rate variability," J. Roy. Soc. Interface, vol. 14, no. 126, 2017, Art. no. 20160513.

[12] C. Stefanadis et al., "A clinical appraisal of left atrial function," Eur. Heart J., vol. 22, no. 1, pp. 22-36, 2001.

[13] P. S. Pagel et al., "Mechanical function of the left atriumnew insights based on analysis of pressure-volume relations and doppler echocardiography," Anesthesiology, vol. 98, no. 4, pp. 975-994, 2003.

[14] M. J. Miller et al., "Right atrial pressure-volume relationships in tricuspid regurgitation," Circulation, vol. 73, no. 4, pp. 799-808, 1986. [Online]. Available: http://circ.ahajournals.org/cgi/doi/10.1161/01.CIR.73.4.799

[15] C. S. Chung et al., "Titin-actin interaction: Pevk-actin-based viscosity in a large animal," BioMed Res. Int., vol. 2011, 2011, Art. no. 310791.

[16] M. R. Rehorn et al., "The passive properties of muscle fibers are velocity dependent," J. Biomech., vol. 47, no. 3, pp. 687-693, 2014.

[17] W. H. Gaasch and T. E. Meyer, "Left ventricular response to mitral regurgitation," Circulation, vol. 118, no. 22, pp. 2298-2303, 2008.

[18] D. H. Harpole et al., "Effects of standard mitral valve replacement on left ventricular function,” Ann. Thoracic Surg, vol. 49, no. 6, pp. 866-874, 1990 .

[19] O. Gaemperli et al., "Real-time left ventricular pressure: Volume loops during percutaneous mitral valve repair with the mitraclip system," Circulation, vol. 5, pp. 1018-1027, 2013.

[20] K. L. Wanderman et al., "Left ventricular performance in mitral regurgitation assessed with systolic time intervals and echocardiography," Amer J. Cardiol., vol. 38, no. 7, pp. 831-835, 1976.

[21] C. Seiler et al., "Quantitation of mitral regurgitation using the systolic/diastolic pulmonary venous flow velocity ratio," J. Amer. Coll. Cardiol., vol. 31, no. 6, pp. 1383-1390, 1998. 